\title{
Analysis of Discussion Forums of a Programming MOOC
}

\author{
Abdelghani Babori ${ }^{1,2}$ \\ ${ }^{1}$ Hassan First University, Settat, Morocco \\ ${ }^{2}$ University of Lille, Lille, France
}

\begin{abstract}
The work presented in this article focuses on the description of the learning process in the MOOC of algorithms and programming. It focuses more specifically on the study of the learning strategies adopted by students by examining discussion forums in a MOOC of algorithms and programming designed for undergraduate students. The results stated that students used two learning strategies to construct algorithmic content: cognitive strategies and social strategies. These results improve the didactic research on the MOOC by providing researchers with elements for the study of learning process in MOOCs.
\end{abstract}

Keywords -distance education, MOOC, didactic, programming

\section{Introduction}

In order to improve the quality of education, several Moroccan universities are already involved in the creation of MOOCs delivered on free platforms Hassan II Casablanca, Hassan First Settat, Cadi Ayyad Marrakech Universities, to name just a few. In this sense, the e-Learning centre of Mohammed V University in Rabat was the first to develop and launch a MOOC in 2014 in Morocco.

DOI: $10.18421 /$ TEM103-56

https://doi.org/10.18421/TEM103-56

Corresponding author: Abdelghani Babori, Hassan First University, Settat, Morocco / University of Lille, Lille, France.

Email: a.babori@uhp.ac.ma

Received: 25 April 2021.

Revised: 10 August 2021.

Accepted: 16 August 2021.

Published: 27 August 2021.

(c) BY-NC-ND (C) 2021 Abdelghani Babori; published by UIKTEN. This work is licensed under the Creative Commons Attribution-NonCommercial-NoDerivs 4.0 License.

The article is published with Open Access at www.temjournal.com
This MOOC, which dealt with the basics of general accounting, was developed for the benefit of 900 students enrolled in the first year of the Faculty of Juridical, Economic and Social Sciences - Souissi (Rabat).

A call for projects was launched in 2017 to select MOOC projects that will feed the MUN platform. Out of a total of 119 projects submitted, 49 MOOC projects including 5 MOOC4 projects developed within the Hassan Premier University of Settat have been selected to be the first MOOCs to be present on MUN at its official launch in June 2019. The "Algorithms: basic concepts and applications", proposed by the university Hassan First, is one of these selected projects which aims to assist students in the development of algorithms. More specifically, this MOOC aims at building step-by-step problemsolving skills by analysing user needs and formulating them through algorithms to translate them into programs.

The MOOC is organized into weeks. There are six obligatory weeks of algorithmic programming and two optional weeks of C programming. Each week consists of several units composed of videos, web pages, quizzes and a discussion forum that

\section{Related Works}

Algorithmic is considered to be a major subject that demands a high level of ability on the part of the students to analyse a problem. It is a difficult subject that has often been a source of problems for novice learners. This problem finds its reasons in both the cognitive aspect (abstract concepts) of the subject that pedagogic strategies adopted in teaching this discipline, where learners are usually passive, demotivated during the learning process, unable to mobilize all the acquired knowledge to resolve new problems [1]. Thus, this MOOC aims to improve the quality of teaching through discussion forums and provide personalized help for students to acquire skills such as autonomy, motivation, time management and son on.

Massive Open Online Courses are interactive training courses given online and open to all by registration. Owing to the MOOCs, it is now possible 
to access the knowledge delivered by the most prestigious institutions around the world. These MOOCs are open to the maximum number of users, generally for free and with no prerequisites [2].

Research on the MOOCs is changing and growing quickly. Several studies focusing on the MOOCs were published since 2008 . We have conducted a literature review on the MOOCs by analyzing research articles published between 2012 and 2018 [3]. This literature review shows that four categories of research objects are identified: learning processes, predictors of retention, learning experiences and the design of the MOOCs. Research questions that deal with the learning process focus on the way in which people self-control their learning, the motivations of participants to learn in a MOOC, the behaviour of the learner in the course. The articles focusing on interactions in the MOOCs address the discussion modalities that describe the learning process in discussion forums, the interactions between learners and the relationship between the different levels of interaction.

Whereas research on the MOOCs focuses more on the learning process, they concentrated much less on content material as an object of research. More specifically, the didactic issues stay minor in this research. Indeed, there isn't yet any research specializing in the appropriation of content material in discussion forums of the MOOCs. Thus, this study will attempt to answer this research question: how do students construct algorithmic content in the discussion forums of the conceived MOOC "Algorithms: Basic Concepts and Applications"?

\section{Methodology}

\subsection{Context}

The 'Algorithmic: Basic concepts and applications MOOC' attracted 1052 registrants. The MOOC goal is to teach participants how to design algorithms and programs. The MOOC is organized into 8 weeks: six obligatory weeks dedicated to algorithmic and two optional weeks of programming in C language. Each week consists of several units composed of videos, web pages, quizzes and a discussion forum that allows discussing the MOOC content. Week zero presents the overall site including an introduction to the MUN (Morocco Digital University) platform and a general introduction to Algorithmic MOOC: Basic Concepts and Applications. The other weeks are organised into themes according to a specified order: from simple to complex tasks. For instance, in the videos or in the quizzes, we start with easy examples and then gradually we tackle complex concepts. Thus, in week one, variables and basic instructions are processed. Week two presents conditions. Week three covers the loops: we proposed a multitude of illustrative examples in order to better understand the progress of each loop. Weeks four and five deal respectively with one-dimensional and twodimensional arrays.

\subsection{Data Collection}

Data was collected between January 2019 and November 2019. The discussion posts were examined to describe the learning strategies adopted by students to apprehend the algorithmic content within and in relation to the MOOC. We selected messages from the MOOC discussion forums. The forum included the following elements: description of the discussion thread, number of discussion posts, date of observation, starting post for each thread and description of each discussion post in the thread.

\subsection{Data Analysis}

An analysis grid to review discussion posts was used. This grid was constructed based on previous research [4], [5]. This research distinguishes between discussion posts related to content and discussion posts unrelated to content (posts that refer to logistical and technical topics). The grid includes multiple-choice as well as open-ended questions and consists of three parts: identification of the discussion thread defined as one topic under discussion by a group (description of the discussion thread, date of observation, number of discussion posts), identification of the posts in each discussion thread (description of the post, type of the post, type of the learning strategy and the identified content (designation, description of the content).

\section{Results}

\subsection{Cognitive Strategies}

Cognitive learning strategies are explicitly manifested by students in the moments when they have interacted in the discussion forums with the pedagogical team or with other students so as to ask or answer questions and make remarks or comments focusing on algorithmic content. Thus, these interactions focused on: the description of the steps of an algorithm using previous knowledge or lived experience (approach to prepare a cake for example); the meaning of a concept (loop, condition); the resolution of a problem (the algorithm of perfect number).

\section{Description of the steps of an algorithm}

The facilitator asks a question in the discussion forum about the steps involved in developing an algorithm. Students describe the steps involved. The facilitator then asks to clarify each step described. 
Facilitator: Question of synthesis: who can summarize for me the steps to follow to solve an algorithmic problem?

Student 1: 1 Problem analysis (input and output variables)

2. Calculation formula

3. Choice of instructions

4. Test the algorithm

Facilitator: Good! If anyone else has any other suggestions?

Student 2: The first step is the pre-analysis (goal of the problem and data) the second step is the analysis the last one is the algorithm.

Facilitator: If you can further develop the steps especially what you mean by the analysis. Thank you!

Student 2: To analyse a problem we ask 3 questions: what is the result we are looking for, i.e., the output of our algorithm? How do we obtain this result, i.e., the processing of our algorithm? What is the necessary information? I.e., the input data of the problem.

Student 3: Hi, e.g., the algorithm for preparing a cake, the input variables are eggs, flour, chocolate, etc., the result is the cake, the treatment is to take the eggs, flour, butter and sugar and mix it all together and put it in the oven for half an hour.

We notice that some students (Student 1, Student 2, Student 3) try to synthesize the steps of an algorithm. After having relaunched, by the facilitator, the ideas expressed by students 1 and 2 by asking them to develop further the answers to the question asked, we can clearly see that student 2 is directly trying to answer the question. Student 3 uses his previous experience (cake preparation) to illustrate the steps involved in analysing a problem. He therefore refers to content constructed in everyday life.

\section{The meaning of concepts}

The student asked about the usefulness of the arrays. The facilitator gives a simple example of an algorithm in which he clearly demonstrates the limit of the use of the variable concept. It continues to specify other uses of arrays.

Student 5: Why can't we use variables instead of complicated arrays?

Facilitator: Hello, the simple example I gave in the Week 4 introduction to one-dimensional arrays is to enter the grades of students in a class. Let's say we want to calculate the overall grades obtained from 100 students Grade 1, Grade 2, Grade 3 .... Grade 100 So how do we do it? With variables we can of course solve this problem by doing the following: Var Note 1, Note 2 and continue like this until Note 100. And continue like this until Write ('give Note 100'); Read (Note 100); Which is very long and not practical. So, the solution is to use a table.

We can see that the cognitive learning strategy of this student consists in asking a question in an attempt to stabilize the notion of arrays (implying the notion of the variable as well). However, it does not react to the explanation provided by the MOOC facilitator.

\section{Mobilizing concepts in an algorithm}

Students ask questions about mobilizing the concepts of loops (For loop) and nested conditions. The facilitator reminds the students of the usefulness of repetitive instructions by explaining in detail how the algorithms mobilizing these two concepts work.

\begin{abstract}
Student 1: Hello, I didn't understand why we didn't use loop for greatest common divisor calculation?

Student 2: Hello, because the number of iterations is not known.

Facilitator: Hello, for the problem of the GCD we did not use the For loop because the number of iterations is not known in advance. Let us recall Euclide's algorithm to calculate the gcd: While the condition: the rest $r$ of the division of a by $b r<>0$ do $a b ; b<-r$; $r<-a \% b$. We saw in the video on repetitive instructions that the instructions $a<-b ; b<-r ; r<-a \% b$; repeat $a$ number of times that we do not know in advance. For example, if $a=10$ and $b=6$, these instructions are repeated exactly twice. $r=10 \% 6=4$ Step 1: $a=6 \quad b=4$ $r=2$ Step 2: $a=4 b=2 r=0$
\end{abstract}

We can see that the cognitive strategies adopted by students in these discussions consisted in asking questions in relation to the tasks to be carried out in order to find solutions to difficulties related to the content (the notions of algorithmics).

\subsection{Social Strategies}

The results of the analysis of the discussion posts show that the students used social learning strategies in terms of interactions between themselves or with the MOOC pedagogical team. More specifically, these interactions focus on the students' point of view on the presentation of the content and its difficulty or the teacher's thanks for the answer to questions. For example, a student expresses his difficulty with the concept of the loop at the beginning and thanks the facilitator at the end:

Student 9: I have to confess that I've been lost since the beginning of the loop instructions. I don't really understand loops especially the perfect number algorithm.

Facilitator: Hello, first a little reminder about the usefulness of repetitive instructions: they are used in any situation where you find a repetition of a block of instructions a certain number of times, instead of using basic instructions (reading for example) you use a loop that saves time in writing an algorithm and executing the associated program....

This social learning strategy is also manifested by some students in the discussion forums by asking for links to download program development tools in $\mathrm{C}$ 
language or expressing difficulties in using a tool integrated in the MOOC platform:

Student 5: Please the dev $c^{++}$installation link. thank you

Student6: Hello, the Blockly Games site is no longer displayed. How to solve this problem? Thank you.

\section{Discussion}

The analysis of the discussion posts shows that the cognitive learning strategies of the students consisted mainly in asking questions to overcome difficulties encountered in the MOOC and that are related to the three concepts of algorithmics: loops, nested conditions and arrays. However, the study reveals shortcomings related to the cognitive strategies of critical thinking such as scrutinizing concepts by discussing the content or considering something in the course to be unsure. Thus, exchanges have taken place, however they only concern a part of the active students. Moreover, we observed that these exchanges are short and that most of the discussion posts focus on questions between students and the pedagogical team in order to obtain an answer to a specific question. In addition, although discussion forums allow interaction despite location or time barriers, according to the interviews, they do not provide a satisfactory user experience. Indeed, the majority of participants stated that the MOOC does not favour group work. These discussion forums are based on asynchronous conversations that do not allow direct collaboration between participants. This is underlined by Dussarps [6] who states that, in distance learning environments, learners wish to meet each other, ideally physically, if not virtually, to discuss the content conveyed by the MOOC. Thus, the integration of synchronous means of communication such as social networks, chat, or other communication tools, would be very useful to promote a deep discussion of the content conveyed.

The data from the discussion forums allow us to identify some learning strategies (cognitive and social strategies) adopted by students but do not inform us of the frequency of resource consultation (consulting videos, web pages, clicking on links, etc.). It would therefore be interesting to combine the different types of the learning analytics in order to determine the factors that could influence the students' learning strategies.

This article which presents the learning strategies adopted by students to learn in the Algorithmic and Programming MOOC differs from other previous studies using discussion posts as data. Indeed, these studies that focused on interactions in MOOCs investigate the different modalities of discussion that characterize learning process in forums [7], [8], the communication modalities that learners prefer: asynchronous or synchronous [9], [10], the interaction processes between users and organizers in a Javascript MOOC [11] or the modes of interaction and their roles in the co-creation of new content [12], [13].

This study shows that students use more cognitive learning strategies than social learning strategies. One explanation for this result may be the students' perception of how they learn. Indeed, we may suppose that throughout their previous experiences, students have developed a representation of what "learning" is by focusing on topics that relate directly to the content in question. Another possible hypothesis lies in the fact that students consider that social learning strategies are less essential to success in MOOCs than cognitive and technical learning strategies that are related to the learning objectives such as completing a learning activity [14].

The results also show that students adopt weakly planning strategy. However, this strategy is important for a successful learning process in MOOCs, which require more autonomy. In fact, the absence of effective planning of learning can result in a disorganized course. Thus, in addition to the content to be taught, learning planning strategies such as to present in the form of a timetable, the learning activities would be useful for registrants in order to organize their learning.

\section{Conclusion}

The results obtained through the analysis of the discussion posts allow us to characterize some learning strategies adopted by the students to appropriate the concepts and procedures of the algorithmic content. However, these strategies concern only a part of the active students in the discussion forums. In addition, the discussion posts highlight only two types of learning strategies: social and cognitive learning strategy. Moreover, even though the platform has discussion forums that can be adopted to work in groups or share resources that prove useful, we found that these discussion forums are rather used by students to communicate with MOOC facilitators in order to find answers to their questions. As a result, we found that communication between students is infrequent.

In this present study, we have identified the learning strategies adopted by students to construct algorithmic content within the MOOC. Nevertheless, the results present some limitations, and could constitute the basis of future research. The results reveal that the strategies related to concentration or time management are not addressed in this research. Indeed, considered as a key to the success of a work, a better management of study time is essential to progress in MOOC [15]. An integration of tools such 
as the electronic agenda, the daily or weekly schedule and the checklist, is thus useful to help participants get motivated and well organized in the MOOC.

As a future work, we therefore propose other implementations on other subjects such as artificial intelligence, machine learning, databases, etc., which pose, in particular, problems for students. Moreover, the description of what students use as learning strategies in the MOOC discussion forums are useful in the sense that researchers may ask: What factors influence the adoption of a learning strategy? What is the link between the learning strategies used by students in the forums and academic success?

\section{References}

[1]. Babori, A., Fassi, H. F., Hariri, A., Bideq, M., \& Zaid, A. (2016, July). Using problem based learning environment to enhance algorithmic problem solving skill. In 2016 Global Summit on Computer \& Information Technology (GSCIT) (pp. 60-65). IEEE.

[2]. Bogdan, R. (2017). Integrating MOOCs in embedded systems blended Courses. BRAIN. Broad Research in Artificial Intelligence and Neuroscience, 8(3), 101107.

[3]. Babori, A., Zaid, A., \& Fassi, H. F. (2019). Research on MOOCs in Major Referred Journals: The Role and Place of Content. International Review of Research in Open and Distributed Learning, 20(3), 221-247.

[4]. Almatrafi, O., Johri, A., \& Rangwala, H. (2018). Needle in a haystack: Identifying learner posts that require urgent response in MOOC discussion forums. Computers \& Education, 118, 1-9.

[5]. Wise, A. F., Cui, Y., Jin, W., \& Vytasek, J. (2017). Mining for gold: Identifying content-related MOOC discussion threads across domains through linguistic modeling. The Internet and Higher Education, 32, 11 28.

[6]. Dussarps, C. (2018). Faut-il (ré) humaniser les MOOC?.Revue française des sciences de l'information et de la communication, (12).
[7]. Gillani, N., \& Eynon, R. (2014). Communication patterns in massively open online courses. The Internet and Higher Education, 23, 18-26.

[8]. Zhang, J., Skryabin, M., \& Song, X. (2016). Understanding the dynamics of MOOC discussion forums with simulation investigation for empirical network analysis

Education, 37(3), 270-286. doi: 10.1080/01587919.2016.1226230

[9]. Li, N., Verma, H., Skevi, A., Zufferey, G., Blom, J., \& Dillenbourg, P. (2014). Watching MOOCs together: investigating co-located MOOC study groups. Distance Education, 35(2), 217-233.

[10]. Zhang, Q., Peck, K. L., Hristova, A., Jablokow, K. W., Hoffman, V., Park, E., \& Bayeck, R. Y. (2016). Exploring the communication preferences of MOOC learners and the value of preference-based groups: Is grouping enough?. Educational Technology Research and Development, 64(4), 809-837.

[11]. Andersen, R., \& Ponti, M. (2014). Participatory pedagogy in an open educational course: challenges and opportunities. Distance education, 35(2), 234249. doi: 10.1080/01587919.2014.917703

[12]. Arora, S., Goel, M., Sabitha, A. S., \& Mehrotra, D. (2017). Learner groups in massive open online courses. American Journal of Distance Education, 31(2), 80-97. doi: 10.1080/08923647.2017.1300461

[13]. Kellogg, S., Booth, S., \& Oliver, K. (2014). A social network perspective on peer supported learning in MOOCs for educators. International Review of Research in Open and Distributed Learning, 15(5), 263-289.

[14]. Cartier, S. (2006). Stratégies d'apprentissage par la lecture rapportées par des élèves en difficulté d'apprentissage de première secondaire en classe de cheminement particulier de formation. Revue des sciences de l'éducation, 32(2), 439-460.

[15]. Mayrand, J. (2017). Utilisation des stratégies d'apprentissage par des étudiants universitaires suite à une formation en efficience cognitive. Thèse de doctorat. Université de Montréal 